\title{
Chern-Simons vortices in supergravity
}

\author{
M. Abou-Zeid* \\ Theoretical Physics Group, The Blackett Laboratory, Imperial College of Science, Technology and Medicine, Prince Consort Road, \\ London SW7 2BWZ, United Kingdom \\ H. Samtleben ${ }^{\dagger}$ \\ Spinoza Insituut, Universiteit Utrecht, Leuvenlaan 4, Postbus 80.195, 3508 TD Utrecht, The Netherlands
}

(Received 10 December 2001; published 2 April 2002)

\begin{abstract}
We study supersymmetric vortex solutions in three-dimensional Abelian gauged supergravity. First, we construct the general $U(1)$-gauged $D=3, N=2$ supergravity whose scalar sector is an arbitrary Kähler manifold with $U(1)$ isometry. This construction clarifies the connection between local supersymmetry and the specific forms of some scalar potentials previously found in the literature-in particular, it provides the locally supersymmetric embedding of the Abelian Chern-Simons Higgs model. We show that the Killing spinor equations admit rotationally symmetric vortex solutions with asymptotically conical geometry which preserve half of the supersymmetry.
\end{abstract}

DOI: 10.1103/PhysRevD.65.085016

PACS number(s): 04.65.+e, 11.27.+d

\section{INTRODUCTION}

In three space-time dimensions, pure Einstein gravity has no local propagating degrees of freedom and is thus topological. The solutions to the field equations are locally flat except at conical singularities at the location of matter sources [1,2]. Moreover, there is a precise sense in which the theory is soluble [3-6]. Similar results hold for cosmological Einstein gravity [7], and for the three-dimensional topological supergravity theories [8,9].

A complete classification of $N$-extended supergravities in three dimensions was given in Ref. [10]. In particular, the geometry of the target manifolds parametrized by the scalar fields is Kähler for $N=2$, quaternionic for $N=3,4$ and symmetric for $N=5,6,8$. For $N=9,10,12,16$, the theories are based on a single supermultiplet and are associated with coset spaces with the exceptional isometry groups $F_{4}, E_{6}$, $E_{7}$, and $E_{8}$, respectively. Recently, a number of maximal $(N=16)$ gauged models with a variety of admissible compact and noncompact gauge groups were constructed in $[11,12]$. In these three-dimensional gauged supergravities, a key role is played by the on-shell duality between the gauge fields and the scalar fields. This is implemented in the Lagrangian by means of a Chern-Simons term for the gauge fields (rather than the usual Yang-Mills term), which ensures that the duality relation is an equation of motion while the gauge fields do not carry physical degrees of freedom.

In this paper we construct the general $U(1)$-gauged nonlinear sigma model coupled to $N=2$ supergravity in three dimensions, and study supersymmetric vortex solutions of this theory. As particular examples, we obtain the supersymmetric embedding of the Abelian Chern-Simons Higgs model coupled to gravity [13-17] and generalizations of the $C P^{n}$

\footnotetext{
*Also at Institut des Hautes Etudes Scientifiques, Le Bois-Marie, 35 route de Chartres, 91440 Bures-sur-Yvette, France. Electronic address: m.abouzeid@ic.ac.uk

${ }^{\dagger}$ Electronic address: H.Samtleben@phys.uu.nl
}

and $\mathrm{CH}^{n}$ models, recently constructed in [18].

The Abelian Higgs model with a Chern-Simons term in three-dimensional flat Minkowski space and its vortex solutions were studied in $[19,20]$ (see also the earlier references therein). This model is of some practical interest because of its relation to the physics of high temperature superconductors, which violate both the $T$ and $P$ symmetries (like the Chern-Simons term) and which often exhibit twodimensional spatial structures. In particular, it was found $[19,20]$ that the model with a specific sixth-order Higgs potential admits topologically stable vortex solutions which satisfy (first order) self-duality, or Bogomol'nyi-type [21] equations. This special Higgs potential has a $U(1)$-symmetric minimum which is degenerate with a symmetry-breaking one, as a result of which it also admits charged nontopological soliton solutions [22]. In Ref. [23], the specific form of this potential was shown to originate from the unique embedding of this model into a globally $N=2$ supersymmetric theory; the Bogomol'nyi bound may be obtained from the superalgebra and is saturated by the supersymmetric solutions.

In the present paper-as a byproduct of our general construction-we give a similar explanation for the origin of the specific eighth-order potential found in the Abelian Chern-Simons Higgs model coupled to gravity. More precisely, it has been found in [13-15] that the Einstein equations and the matter field equations of this model can be recast into a set of self-duality equations for a specific eighth-order choice of the Higgs potential which reduces to the sixth-order potential of the flat space model when the Newton gravitational coupling constant is set to zero. We show that this is the unique potential which may be embedded into a locally supersymmetric theory, with the Bogomol'nyi-type equations descending from the Killing spinor equations of this underlying supergravity. This in particular allows us to address the stability of the vortex solutions studied in [13-17].

In the second part of the paper we study supersymmetric vortex solutions of the general $U(1)$-gauged supergravity 
with a single complex scalar field. We show that with a rotationally symmetric ansatz, the Killing spinor equations reduce to a set of four first-order differential equations. This generalizes the results of $[15,17]$, where these equations were found by the Bogomol'nyi-type arguments mentioned above, to arbitrary Kähler manifolds. Identifying an integral of motion, we show that after suitable redefinition of coordinates, these equations may further be reduced to a single secondorder differential equation. For a given vortex number, it has a unique solution with regular asymptotics, from which all the original fields may be restored. It represents a rotationally symmetric, finite energy, topologically stable vortex solution, preserving one-half of the supersymmetry.

Another motivation for the study of locally supersymmetric theories in three dimensions and their solutions stems from an observation made by Witten [24]: in three dimensions, the cosmological constant of the vacuum can be exactly zero because of local supersymmetry, yet the spectrum of excited states may not exhibit the usual Bose-Fermi mass degeneracy because for non-zero energy states the supercharges are defined in conical space-times. A realization of this mechanism in the $N=2$ supersymmetric Abelian Maxwell-Higgs model coupled to gauged three-dimensional supergravity was exhibited in [25], and studied further e.g., in Refs. [26-28]. The Abelian gauged $N=2$ supergravities and their half supersymmetric vortex solutions constructed in the present paper provide additional examples of this mechanism. As in [25,27], the covariantly constant spinors required to define these supersymmetries exist by virtue of a cancellation between the Aharonov-Bohm phase and the phase associated with the holonomy of the spin connection. However, the same mechanism as in [25] prevents the existence of normalizable covariantly constant spinors associated with the other half of the supersymmetry transformations, and hence Bose-Fermi degeneracy is absent in the soliton spectrum. Of course, it remains to be seen whether a locally supersymmetric four-dimensional theory with zero cosmological constant but without the phenomenologically unviable Bose-Fermi degeneracies can be constructed along the lines suggested in [24].

The plan of this paper is as follows. In Sec. II we construct the general $U(1)$-gauged $N=2$ supergravity by deforming the three-dimensional sigma model of Ref. [10], whose target space is an arbitrary Kähler manifold with $U(1)$ isometry. We show that our results reduce to those of [18] when the target spaces are the homogeneous spaces $C P^{n}$ and $C H^{n}$ (and, moreover, include a possible extension by Fayet-Iliopoulos terms), while for the complex plane they give the supersymmetric embedding of the Abelian ChernSimons Higgs model coupled to gravity. In Sec. III we study the Killing spinor equations of the general model with a single complex scalar field and we find that they reduce to a set of four first-order differential equations. We then show that they admit a unique solution with the prescribed (regular) asymptotics and give some numerical examples. We close with a summary and comments on possible applications.

\section{II. $U(1)$-GAUGED $D=3, N=2$ SUPERGRAVITY}

We start this section by reviewing the Lagrangian and transformations rules for a non-linear sigma model coupled to $N=2$ supergravity. This mainly serves to set our notation and conventions; the reader is referred to [10] for full results and a detailed discussion. Assuming an $U(1)$ isometry of the Kähler potential, we apply the standard Noether procedure to obtain the general $U(1)$-gauged $N=2$ supergravity. In four dimensions, analogous supergravity theories have been studied in [29-31]. We then evaluate the general formulas in several examples.

\section{A. $D=3, N=2$ supergravity and Kähler geometry}

The gravity multiplet of the ungauged $N=2$ supergravity in three dimensions consists of a dreibein $e_{\mu}{ }^{a}$ and two gravitini which we assemble into one complex spinor $\psi_{\mu}$. The matter sector is given by $p$ copies of the $N=2$ scalar multiplet, each consisting of two real scalars and fermions. Again we use complex notation $\left(\phi^{\alpha}, \bar{\phi}^{\bar{\alpha}}\right)$ and $\left(\lambda^{\alpha}, \bar{\lambda}^{\bar{\alpha}}\right)$ $(\alpha=1, \ldots, p)$, respectively. The scalar fields parametrize a Kähler manifold of real dimension $2 p$, characterized by its Kähler potential $K\left(\phi^{\alpha}, \bar{\phi}^{\bar{\alpha}}\right)$.

The $N=2$ locally supersymmetric Lagrangian is given by

$$
\begin{aligned}
\mathcal{L}^{S}= & \frac{1}{4} e R+\varepsilon^{\mu \nu \rho} \bar{\psi}_{\mu} \mathcal{D}_{\nu} \psi_{\rho}-e G_{\alpha \bar{\alpha}}(\phi, \bar{\phi})\left(\partial_{\mu} \phi^{\alpha} \partial^{\mu} \bar{\phi}^{\bar{\alpha}}\right. \\
& \left.+\bar{\lambda}^{\bar{\alpha}} \gamma^{\mu} \mathcal{D}_{\mu} \lambda^{\alpha}\right)+e G_{\alpha \bar{\alpha}}(\phi, \bar{\phi}) \bar{\lambda}^{\bar{\alpha}} \gamma^{\mu} \gamma^{\nu} \psi_{\mu} \partial_{\nu} \phi^{\alpha} \\
& +e G_{\alpha \bar{\alpha}}(\phi, \bar{\phi}) \bar{\psi}_{\mu} \gamma^{\nu} \gamma^{\mu} \lambda^{\alpha} \partial_{\nu} \bar{\phi}^{\bar{\alpha}},
\end{aligned}
$$

up to terms quartic in the fermions. Here, $G_{\alpha \bar{\alpha}}(\phi, \bar{\phi})$ denotes the Kähler metric $G_{\alpha \bar{\alpha}}(\phi, \bar{\phi})=\partial_{\alpha} \partial_{\alpha} K(\phi, \bar{\phi})$. We use $2 \times 2$ matrices $\gamma^{a}$ for the $S O(2,1)$ Dirac algebra, with $\gamma^{a} \gamma^{b}$ $=\eta^{a b}-\varepsilon^{a b c} \gamma_{c}$; the charge conjugation matrix is $\gamma^{0}$. Our metric has signature $(-++)$, and $\varepsilon^{012}=1$. A convenient representation is $\gamma^{0}=i \sigma^{3}, \gamma^{1}=-\sigma^{2}$ and $\gamma^{2}=-\sigma^{1}$. The sign of the Newton gravitational coupling, which in three dimensions is not physically fixed, is taken to be positive. The Kähler covariant derivatives acting on the fermions are

$D_{\mu} \psi_{\nu}=\left(\nabla_{\mu}-Q_{\alpha} \partial_{\mu} \phi^{\alpha}+Q_{\bar{\alpha}} \partial_{\mu} \bar{\phi}^{\bar{\alpha}}\right) \psi_{\nu}$,

$D_{\mu} \lambda^{\beta}=\left(\nabla_{\mu}-Q_{\alpha} \partial_{\mu} \phi^{\alpha}+Q_{\alpha} \partial_{\mu} \bar{\phi}^{\bar{\alpha}}\right) \lambda^{\beta}+\Gamma_{\alpha \gamma}^{\beta} \partial_{\mu} \phi^{\alpha} \lambda^{\gamma}$,

with spin-, $S O(2)-$, and Kähler connections

$$
\begin{gathered}
\nabla_{\mu}=\partial_{\mu}+\frac{1}{4} \omega_{\mu}^{a b} \gamma_{a b}, \\
Q_{\alpha}(\phi, \bar{\phi})=\frac{1}{2} \partial_{\alpha} K(\phi, \bar{\phi}), \\
Q_{\alpha}^{-}(\phi, \bar{\phi})=\frac{1}{2} \partial_{\bar{\alpha}} K(\phi, \bar{\phi}), \\
\Gamma_{\beta \gamma}^{\alpha}(\phi, \bar{\phi})=G^{\alpha \bar{\alpha}}(\phi, \bar{\phi}) \partial_{\beta} G_{\gamma \bar{\alpha}}(\phi, \bar{\phi}) .
\end{gathered}
$$


Up to cubic terms, the supersymmetry transformations which leave the Lagrangian (1) invariant are given by

$$
\begin{aligned}
\delta e_{\mu}{ }^{a} & =\bar{\epsilon} \gamma^{a} \psi_{\mu}-\bar{\psi}_{\mu} \gamma^{a} \epsilon, \\
\delta \psi_{\mu} & =D_{\mu} \epsilon=\left(\nabla_{\mu}-Q_{\alpha} \partial_{\mu} \phi^{\alpha}+Q_{\alpha} \partial_{\mu} \bar{\phi}^{\bar{\alpha}}\right) \epsilon, \\
\delta \phi^{\alpha} & =\bar{\epsilon} \lambda^{\alpha}, \\
\delta \lambda^{\alpha} & =\partial_{\mu} \phi^{\alpha} \gamma^{\mu} \epsilon,
\end{aligned}
$$

where $\epsilon$ is a complex spinor.

\section{B. Kähler transformations and isometries}

The sigma model geometry of Sec. II A is clearly invariant under the Kähler transformations

$$
K(\phi, \bar{\phi}) \rightarrow K(\phi, \bar{\phi})+F(\phi)+\bar{F}(\bar{\phi}),
$$

while the potential $Q_{\alpha}$ transforms as

$$
Q_{\alpha} \rightarrow Q_{\alpha}+\frac{1}{2} \partial_{\alpha} F(\phi) .
$$

As in higher dimensions $[29,30]$, the Lagrangian (1) is invariant under these transformations, provided the fermionic fields simultaneously transform as

$$
\psi_{\mu} \rightarrow \exp (i \Im F) \psi_{\mu}, \quad \lambda \rightarrow \exp (i \Im F) \lambda .
$$

Moreover, we assume that the Kähler potential possesses an $U(1)$ isometry generated by the vector field

$$
\xi=i\left(\phi^{\alpha} \partial_{\alpha}-\bar{\phi}^{\bar{\alpha}} \partial_{\bar{\alpha}}\right)
$$

i.e., $\xi$ generates a Kähler transformation

$$
\mathcal{L}_{\xi} K=i\left(\phi^{\alpha} \partial_{\alpha}-\bar{\phi}^{\bar{\alpha}} \partial_{\alpha}\right) K=f(\phi)+\bar{f}(\bar{\phi}) .
$$

The Lagrangian (1) is then invariant under the global $U(1)$ :

$$
\begin{aligned}
& \delta \phi^{\alpha}=i q \phi^{\alpha}, \\
& \delta \psi_{\mu}=i q \Im f \psi_{\mu}, \\
& \delta \lambda^{\alpha}=i q(1+\Im f) \lambda^{\alpha},
\end{aligned}
$$

where the functional parameter $f$ is defined in terms of the Kähler potential $K$ by Eq. (9). Observe that Eq. (9) determines the holomorphic function $f$ only up to a constant imaginary shift

$$
f \rightarrow f+i c,
$$

with $c$ real.

\section{The gauged Lagrangian and transformation rules}

We proceed to gauge the Abelian Kähler isometry (10) of the locally supersymmetric Lagrangian (1). As in the maximally supersymmetric theories $[11,12]$, this requires the cou- pling to a Chern-Simons gauge potential $A_{\mu}$ with Abelian field strength $F_{\mu \nu}=2 \partial_{[\mu} A_{\nu]}$ via

$$
\mathcal{L}^{C S}=-\frac{1}{8} g \varepsilon^{\mu \nu \rho} A_{\mu} F_{\nu \rho} .
$$

Note that this term is topological and hence does not introduce new propagating degrees of freedom in the gauged theory. The derivatives in Eq. (2) are gauge covariantized according to

$$
\begin{aligned}
\mathcal{D}_{\mu} \phi^{\alpha} \equiv & \left(\partial_{\mu}+i g A_{\mu}\right) \phi^{\alpha}, \\
\mathcal{D}_{\mu} \psi_{\nu} \equiv & {\left[\nabla_{\mu}-Q_{\alpha} \mathcal{D}_{\mu} \phi^{\alpha}+Q_{\alpha} \mathcal{D}_{\mu} \bar{\phi}^{\bar{\alpha}}+i g(\Im f) A_{\mu}\right] \psi_{\nu} } \\
= & \left(\nabla_{\mu}-Q_{\alpha} \partial_{\mu} \phi^{\alpha}+Q_{\alpha} \partial_{\mu} \bar{\phi}^{\alpha}+i g C A_{\mu}\right) \psi_{\nu}, \\
\mathcal{D}_{\mu} \lambda^{\beta} \equiv & {\left[\nabla_{\mu}-Q_{\alpha} \partial_{\mu} \phi^{\alpha}+Q_{\alpha}^{-} \partial_{\mu} \bar{\phi}^{\bar{\alpha}}+i g(1+C) A_{\mu}\right] \lambda^{\beta} } \\
& +\Gamma_{\alpha \gamma}^{\beta} \mathcal{D}_{\mu} \phi^{\alpha} \lambda^{\gamma},
\end{aligned}
$$

where we have defined the real Kähler invariant function

$$
C=-Q_{\alpha} \phi^{\alpha}-Q_{\alpha} \bar{\phi}^{\bar{\alpha}}-\frac{1}{2} i(f-\bar{f}) .
$$

The latter transforms under the constant shifts (11) as

$$
C \rightarrow C+c .
$$

As will be seen shortly, this function is intimately related to the superpotential of the gauged theory. The freedom in picking a constant $c$ in Eq. (15) can then be related to the existence of the Fayet-Iliopoulos term.

As usual, the $g$-dependent terms introduced above give rise to extra terms in the supersymmetry variation of Eq. (1) according to

$$
\begin{aligned}
{\left[\mathcal{D}_{\mu}, \mathcal{D}_{\nu}\right] \psi_{\rho}=} & \frac{1}{4} R_{\mu \nu}{ }^{a b} \gamma_{a b} \psi_{\rho}+2 G_{\alpha \bar{\alpha}} \mathcal{D}_{[\mu} \phi^{\alpha} \mathcal{D}_{\nu]} \bar{\phi}^{\bar{\alpha}} \psi_{\rho} \\
& +i g C F_{\mu \nu} \psi_{\rho}
\end{aligned}
$$

In order for these terms to vanish, extra Yukawa-type bilinear fermionic terms and a scalar potential $V(\phi, \bar{\phi})$ must be added:

$$
\begin{aligned}
\mathcal{L}^{Y}= & -e g \bar{\psi}_{\mu} \gamma^{\mu \nu} \psi_{\nu} B-e g \bar{\lambda}^{\bar{\alpha}} \lambda^{\alpha} S_{\alpha \bar{\alpha}}+e g C\left(\bar{\psi}_{\mu} \gamma^{\mu} \lambda^{\alpha} S_{\alpha}\right. \\
& \left.-\bar{\lambda}^{\bar{\alpha}} \gamma^{\mu} \psi_{\mu} S_{\alpha}^{-}\right), \\
\mathcal{L}^{V}= & e g^{2} V .
\end{aligned}
$$

Here, the functionals $B(\phi, \bar{\phi})$ and $V(\phi, \bar{\phi})$ are real scalars, while the vector functionals $S_{\alpha}(\phi, \bar{\phi})=S_{\alpha}^{-}(\phi, \bar{\phi})$ and the tensor functionals $S_{\alpha \bar{\beta}}(\phi, \bar{\phi})$ are complex. Their dependence on the scalars $\phi, \bar{\phi}$ will be specified below. 
The supersymmetry variations are likewise modified by g-dependent contributions:

$$
\begin{aligned}
\delta e_{\mu}{ }^{a}= & \bar{\epsilon} \gamma^{a} \psi_{\mu}-\bar{\psi}_{\mu} \gamma^{a} \epsilon, \\
\delta \psi_{\mu}= & \mathcal{D}_{\mu} \epsilon-g B \gamma_{\mu} \epsilon, \\
\delta \phi^{\alpha}= & \bar{\epsilon} \lambda^{\alpha}, \\
\delta \lambda^{\alpha}= & \mathcal{D}_{\mu} \phi^{\alpha} \gamma^{\mu} \epsilon-g G^{\alpha \bar{\alpha}} C S_{\alpha}^{-} \epsilon, \\
\delta A_{\mu}= & -2 i\left(\bar{\epsilon} \psi_{\mu}-\bar{\psi}_{\mu} \epsilon\right) C \\
& +2 i G_{\alpha \bar{\alpha}}\left(\phi^{\alpha} \bar{\lambda}^{\bar{\alpha}} \gamma_{\mu} \epsilon+\bar{\phi}^{\bar{\alpha}} \bar{\epsilon} \gamma_{\mu} \lambda^{\alpha}\right) .
\end{aligned}
$$

A straightforward calculation shows that-modulo higher order fermionic terms which presumably remain unchanged as in the maximal theories of $[11,12]$ — the full Lagrangian,

$$
\mathcal{L}=\mathcal{L}^{S}+\mathcal{L}^{C S}+\mathcal{L}^{Y}+\mathcal{L}^{V}
$$

is invariant under Eq. (18) if the functionals $B, S_{\alpha}, S_{\alpha \bar{\alpha}}$ satisfy the following set of consistency relations:

$$
\begin{aligned}
\partial_{\alpha} B & =-C S_{\alpha}, \\
S_{\alpha} & =2 G_{\alpha \bar{\alpha}} \bar{\phi}^{\bar{\alpha}}, \\
D_{\beta} S_{\alpha} & \equiv \partial_{\beta} S_{\alpha}-\Gamma_{\alpha \beta}^{\gamma} S_{\gamma}=0, \\
S_{\alpha \bar{\beta}} & =B G_{\alpha \bar{\beta}}-S_{\alpha} S_{\bar{\beta}}+C \partial_{\bar{\beta}} S_{\alpha},
\end{aligned}
$$

while the potential is given by

$$
\begin{aligned}
V & =2 B^{2}-G^{\alpha \bar{\alpha}} C^{2} S_{\alpha} S_{\bar{\alpha}} \\
& =2 B^{2}-G^{\alpha \bar{\alpha}} \partial_{\alpha} B \partial_{\alpha}^{-} B .
\end{aligned}
$$

It is easily checked that the system (20) is consistent. The general solution to these equations takes the form

$$
\begin{aligned}
B & =C^{2}+b, \\
S_{\alpha} & =-2 \partial_{\alpha} C=2 G_{\alpha \bar{\alpha}} \bar{\phi}^{\bar{\alpha}}, \\
S_{\alpha \bar{\beta}} & =B G_{\alpha \bar{\beta}}-S_{\alpha} S_{\bar{\beta}}-2 C \partial_{\alpha} \partial_{\bar{\beta}} C,
\end{aligned}
$$

where $b$ is an arbitrary constant real parameter.

This completes the construction of a family of $N=2 \mathrm{su}$ persymmetric gauged Lagrangians parametrized by a Kähler manifold and two real numbers $b$ and $c$. In particular, the constant shifts of $C$ by $c$ correspond to the presence of a Fayet-Iliopoulos term [30]. Note that according to Eq. (21) $B=C^{2}+b$ is the superpotential for $V$. In the limit $g \rightarrow 0$, one recovers the ungauged theory (1).

The gauged Lagrangian (19) is still invariant under general Kähler transformations (5), (7), under which the functional parameter $f$ changes as

$$
f \rightarrow f+i \phi^{\alpha} \partial_{\alpha} F
$$

\section{Examples}

In this section we consider some special cases of the Abelian gauged Lagrangian constructed in the preceding section. In particular, this will reproduce and explain the form of the eighth order potential of the Abelian Chern-Simons Higgs model coupled to gravity, which was previously derived in [13-17] by Bogomol'nyi-type arguments. Moreover, we reproduce the gauged theories constructed in [18] and their generalization by Fayet-Iliopoulos terms.

In this paper we will mainly be interested in the case $p=1$, i.e. that of a single complex scalar field. Furthermore, we will restrict ourselves to Kähler manifolds for which $f$ as defined in Eq. (9) is an imaginary constant $f=i C_{0}$, i.e. $K$ is a function of $R=|\phi|$ only. We then use the notations

$$
\begin{aligned}
& \phi \equiv \phi^{1}, \\
& Q \equiv Q_{\overline{1}}=\frac{\phi}{4 R} K^{\prime}, \\
& G \equiv G_{1 \overline{1}}=\frac{1}{4 R}\left(R K^{\prime \prime}+K^{\prime}\right),
\end{aligned}
$$

while for the Yukawa tensors and the potential we find

$$
\begin{aligned}
& B=C^{2}+b, \quad C=-\frac{1}{2} R K^{\prime}+C_{0}, \\
& S \equiv S_{\overline{1}}=\frac{\phi}{2 R}\left(R K^{\prime \prime}+K^{\prime}\right), \\
& \mathbf{S} \equiv S_{1 \overline{1}}=B G-|S|^{2}-C C^{\prime \prime}, \\
& V=2 B^{2}-4 G R^{2} C^{2} .
\end{aligned}
$$

Here, $K^{\prime}, K^{\prime \prime}$, etc. denote the derivatives of $K$ with respect to $R=\phi$.

The simplest example in this class of models is the complex plane, with Kähler potential $K(R)=R^{2}$. The above formulas then reduce to

$$
\begin{aligned}
G= & 1, \\
C= & -R^{2}+C_{0}, \\
V_{0}= & -4 R^{2}\left(R^{2}-\eta^{2}\right)^{2} \\
& +2\left(R^{4}-2 R^{2} \eta^{2}+\phi_{\infty}^{2} \eta^{4}\right)^{2},
\end{aligned}
$$

where we have set

$$
\eta^{2}=C_{0}, \quad \phi_{\infty}^{2}=\frac{b+C_{0}^{2}}{C_{0}^{2}} .
$$

This precisely reproduces the eighth order potential of the Abelian Chern-Simons Higgs model coupled to gravity which was derived in [13-17] by requiring the existence of Bogomol'nyi-type equations. What we have shown here is that this form of the potential is naturally explained by su- 
persymmetry: it is the unique potential (depending on two arbitrary parameters $\eta$ and $\phi_{\infty}$ ) which allows the embedding into an $N=2$ locally supersymmetric theory (19). In the following section, we will see in more detail that the Bogomol'nyi-type equations found in [15] indeed descend from the supergravity Killing spinor equations.

For other examples we may consider the Kähler potentials

$$
K_{\varepsilon}(R)=\frac{\varepsilon}{a^{2}} \ln \left(1+\varepsilon R^{2}\right)
$$

The cases $\varepsilon=+1$ and $\varepsilon=-1$ correspond to the coset spaces $S^{2}=S U(2) / U(1)$ and $H^{2}=S U(1,1) / U(1)$, respectively; the constant $a$ denotes the characteristic curvature of these manifolds. With the particular choice of parameters

$$
C_{0}=\frac{\varepsilon}{2 a^{2}}, \quad b=0,
$$

the above formulas reduce to

$$
\begin{aligned}
G_{\varepsilon} & =\frac{1}{a^{2}\left(1+\varepsilon R^{2}\right)^{2}}, \\
C_{\varepsilon} & =\frac{\varepsilon}{2 a^{2}} \frac{1-\varepsilon R^{2}}{1+\varepsilon R^{2}}, \\
V_{\varepsilon} & =\frac{\left.\left(1-\varepsilon R^{2}\right)^{2}\left[\left(1-\varepsilon R^{2}\right)^{2}-8 a^{2} R^{2}\right)\right]}{8 a^{8}\left(1+\varepsilon R^{2}\right)^{4}},
\end{aligned}
$$

which upon setting $g=4 m a^{4}$ and rescaling the vector field $A_{\mu} \rightarrow-\varepsilon g^{-1} A_{\mu}$ precisely reproduces the two models studied in detail in Ref. [18]. For a complete translation between the notation of [18] and that of the present paper, we note that the matter fermions differ by rescaling with a vielbein living on the Kähler manifold, which for $p=1$ simply reduces to $\sqrt{G}$; cf. [10] for further details. Likewise, for higher $p$ and for particular choices of the Kähler manifold, Eqs. (19)-(22) reproduce the $C P^{p}$ and $C H^{p}$ models of Ref. [18].

Our general construction, furthermore, yields a straightforward generalization of Eq. (29) by introducing a FayetIliopoulos term, i.e. leaving $C_{0}$ as a free parameter

$$
C_{0}=\frac{\eta^{2}}{a^{2}\left(1+\varepsilon \eta^{2}\right)}
$$

we find

$$
\begin{aligned}
& G_{\varepsilon}=\frac{1}{a^{2}\left(1+\varepsilon R^{2}\right)^{2}}, \\
& C_{\varepsilon}=\frac{\eta^{2}-R^{2}}{a^{2}\left(1+\varepsilon R^{2}\right)\left(1+\varepsilon \eta^{2}\right)},
\end{aligned}
$$

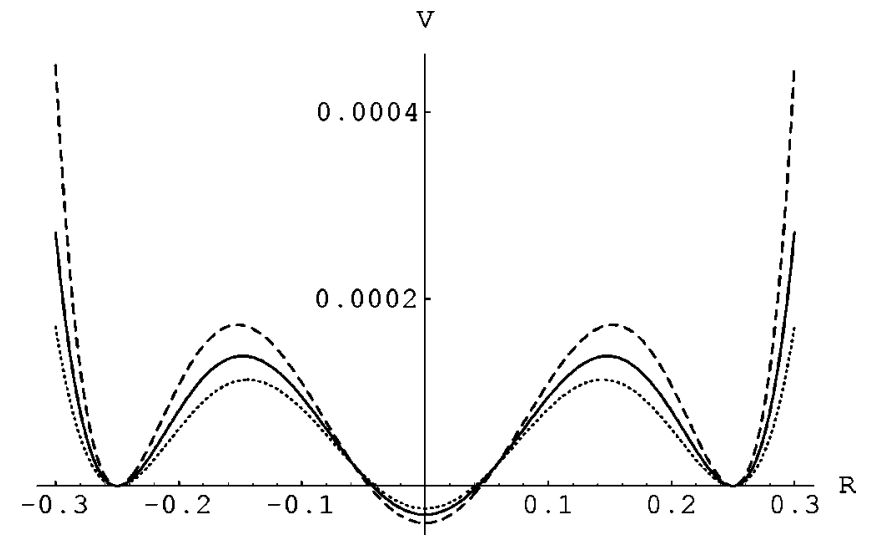

FIG. 1. Scalar potentials $V_{0}$ (solid), $V_{+}$(dotted), $V_{-}$(dashed), from Eqs. (26), (31): vacua at $R=0, R= \pm \eta$.

$$
V_{\varepsilon}=\frac{2\left(R^{2}-\eta^{2}\right)^{2}\left[\left(R^{2}-\eta^{2}\right)^{2}-2 a^{2} R^{2}\left(1+\varepsilon \eta^{2}\right)^{2}\right]}{a^{8}\left(1+\varepsilon R^{2}\right)^{4}\left(1+\varepsilon \eta^{2}\right)^{4}}
$$

These potentials have Minkowski vacua at $R= \pm \eta$. The particular choices in Eq. (29) correspond to $\eta^{2}=\varepsilon$. In the following, we will mainly be interested in the models with potentials given by Eqs. (26) and (31). As an illustration, in Figs. 1 and 2 we depict these potentials for the particular values of parameters $a=1, \eta=1 / 4$. Their behavior for 0 $<R<\eta$ is of similar form, exhibiting a $U(1)$-symmetric AdS vacuum at $R=0$ and symmetry breaking Minkowski vacua at $R= \pm \eta$. Their global behavior, however, differs drastically with $V_{+}$being bounded whereas $V_{0}$ and $V_{-}$become singular at infinite and finite $R$, respectively. We will see in the next section that all these potentials support supersymmetric vortex solutions interpolating between $R=0$ and $R=\eta$.

\section{SUPERSYMMETRIC VORTICES}

In this section we derive a set of first order differential equations for rotationally symmetric, supersymmetric field configurations and show that they admit regular vortex solutions. We restrict ourselves to models with a single scalar

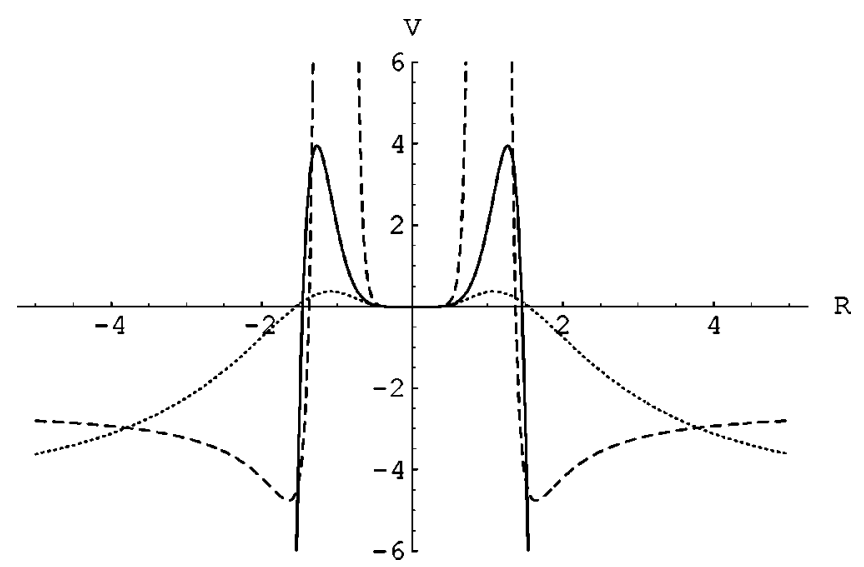

FIG. 2. Scalar potentials $V_{0}$ (solid), $V_{+}$(dotted), $V_{-}$(dashed), from Eqs. (26), (31): global behavior. 
field $p=1$ and a constant value of $f$ in Eq. (9), i.e. to Kähler potentials depending on the absolute value $|\phi|$ only. It would be interesting to generalize this construction to Kähler potentials with an arbitrary holomorphic $f=f(\phi)$; however, this necessitates a more general ansatz for the $\theta$ dependence of the fields than will be considered here. For the complex scalar $\phi$, we make the following time independent and rotationally symmetric ansatz:

$$
\phi=R(r) e^{-i n \theta} .
$$

For the vector field $A_{\mu}$ we choose the gauge in which

$$
A_{r}=0, \quad A_{\theta}=P(r)+\frac{n}{g}, \quad A_{t}=W(r) .
$$

Together with the boundary conditions at the origin and at infinity discussed in detail in Sec. III C, Eqs. (32) and (33) constitute our ansatz for the static and rotationally symmetric $n$-vortex.

For the three-dimensional metric, we take the (stationary and rotationally symmetric) parametrization

$$
d s^{2}=-d t^{2}+2 l(r) \sinh \chi(r) d t d \theta+l^{2}(r) d \theta^{2}+d r^{2}
$$

The Chern-Simons term in the Lagrangian induces the first order duality equations

$$
\varepsilon^{\mu \nu \rho} F_{\nu \rho}=8 i e G\left(\bar{\phi} \mathcal{D}^{\mu} \phi-\phi \mathcal{D}^{\mu} \bar{\phi}\right),
$$

relating the vector and the scalar field. With the ansatz (32)(34), two of these equations take the form

$$
\begin{aligned}
& l \cosh \chi \partial_{r} W=-4 g G R^{2}(P+l \sinh \chi W), \\
& l \cosh \chi \partial_{r} P=-4 g G R^{2}\left(l^{2} W-l \sinh \chi P\right),
\end{aligned}
$$

while the third one $(\mu=r)$ is identically satisfied.

\section{A. Killing spinors}

For supersymmetric vortices we seek solutions to the Killing spinor equations

$$
\delta_{\epsilon} \psi_{\mu} \stackrel{!}{=} 0, \quad \delta_{\epsilon} \lambda \stackrel{!}{=} 0
$$

The transformation of the matter fermions is given by

$$
\delta_{\epsilon} \lambda=\left(\mathcal{D}_{\mu} \phi \gamma^{\mu}-2 g C \phi\right) \epsilon=0,
$$

together with the complex conjugate equation. The existence of nontrivial solutions to this equation implies that

$$
(2 g C \phi)^{2}=\mathcal{D}_{\mu} \phi \mathcal{D}^{\mu} \phi,
$$

which yields

$$
4 C^{2}=W^{2}+\left(\frac{\partial_{r} R}{g R}\right)^{2}-\frac{1}{\cosh ^{2} \chi}\left(W \sinh \chi+\frac{P}{l}\right)^{2} .
$$

This equation is solved, e.g. by setting

$$
\begin{aligned}
W & =-2 C, \\
\frac{\partial_{r} R}{R} & =-\frac{g}{\cosh \chi}\left(W \sinh \chi+\frac{P}{l}\right) .
\end{aligned}
$$

Note that Eq. (24) implies the relation $\partial_{R} C=-2 R G$. Utilizing this, the ansatz (40) is shown to be compatible with the duality equations (36). Substituting Eq. (40) back into Eq. (38), the latter equation can be factorized as

$$
\left(2 l C-i P \gamma^{1}\right)\left(\cosh \chi+i \gamma^{0}-\sinh \chi \gamma^{2}\right) \epsilon=0 .
$$

It is straightforward to check that the solution to this projector equation is given by

$$
\epsilon=\left[\cosh \left(\frac{1}{2} \chi\right)+\sinh \left(\frac{1}{2} \chi\right) \gamma^{2}\right] \epsilon_{0},
$$

with $\quad\left(1+i \gamma^{0}\right) \epsilon_{0}=0$

It remains to study the first equation of (37), i.e. the requirement of vanishing transformation of the gravitini, which reads explicitly

$$
\left[\nabla_{\mu}-\bar{Q} \partial_{\mu} \phi+Q \partial_{\mu} \bar{\phi}+i g A_{\mu} C-g B \gamma_{\mu}\right] \epsilon=0
$$

For $\mu=\theta$ this yields

$$
\begin{aligned}
\partial_{\theta} \epsilon= & -\left(i g P C+\frac{n}{2}(f-\bar{f})\right) \epsilon-\frac{1}{2} l^{\prime} \gamma^{0} \epsilon \\
& -\frac{1}{4}\left(l \chi^{\prime}-l^{\prime} \tanh \chi-4 g B l\right) \gamma^{1} \epsilon,
\end{aligned}
$$

where primes now denote derivatives with respect to the radial variable $r: \chi^{\prime} \equiv \partial_{r} \chi$, etc. Utilizing the projection (41) to eliminate $\gamma^{1} \epsilon$, this equation can be equivalently written as

$$
\begin{aligned}
\partial_{\theta} \epsilon= & -i\left(g P C+\frac{1}{2} n C_{0}\right) \epsilon+\frac{i}{4 \sinh \chi}\left(l \chi^{\prime}-l^{\prime} \tanh \chi\right. \\
& -4 g B l) \epsilon-\frac{1}{4} \operatorname{coth} \chi\left(l \chi^{\prime}+l^{\prime} \tanh \chi-4 g B l\right) \gamma^{0} \epsilon .
\end{aligned}
$$

The term in $\gamma^{0} \epsilon$ must vanish separately, which gives an equation for the metric coefficients, viz.

$$
l \chi^{\prime}+l^{\prime} \tanh \chi=4 g B l \text {. }
$$

Substituting this back into Eq. (45), we find

$$
\partial_{\theta} \epsilon=-i\left(g P C+\frac{1}{2} n C_{0}+\frac{l^{\prime}}{2 \cosh \chi}\right) \epsilon,
$$

which is solved by a separation of variables such that

$$
l^{\prime}=-\left(2 g P C+n C_{0}+2 k\right) \cosh \chi, \quad \partial_{\theta} \epsilon=i k \epsilon,
$$


where $k$ is an arbitrary real parameter. The Killing spinor equation (43) for $\mu=t$ may be treated similarly. Separating variables and utilizing (46) and the projection (41), Eq. (43) reduces to

$$
W=-\frac{2 B}{C}-\frac{k^{\prime}}{g C}, \quad \partial_{t} \epsilon=i k^{\prime} \epsilon,
$$

which coincides with Eq. (40) provided that $k^{\prime}=-2 g b$ and thus determines the time dependence of the spinor. Finally there remains $\mu=r$, for which Eq. (43) takes the form

$$
\partial_{r} \epsilon=\frac{1}{2} \chi^{\prime} \gamma^{2} \epsilon
$$

This equation is indeed satisfied by the ansatz (42), provided $\partial_{r} \epsilon_{0}=0$. Together with Eq. (42) and (47), this implies that the Killing spinor is given by

$$
\epsilon(t, \theta, r)=e^{i k \theta} e^{-2 i g b t}\left[\cosh \left(\frac{1}{2} \chi\right)+\sinh \left(\frac{1}{2} \chi\right) \gamma^{2}\right] \epsilon_{0},
$$

with a constant spinor $\epsilon_{0}$ satisfying

$$
\left(1+i \gamma^{0}\right) \epsilon_{0}=0 .
$$

The field configurations which solve Eqs. (40), (46), and (47) preserve half of the space-time supersymmetry; the corresponding covariantly Killing spinor is given by Eq. (49).

\section{B. The Bogomol'nyi equations}

To summarize, we have shown that with the ansatz (32)(34), the duality and the Killing spinor equations reduce to the following set of first order differential equations:

$$
\begin{aligned}
\partial_{r} R & =g R\left(2 \tanh \chi C-\frac{1}{l \cosh \chi} P\right), \\
\partial_{r} P & =4 g G R^{2}\left(\frac{2 l}{\cosh \chi} C+\tanh \chi P\right), \\
\partial_{r} l & =\left(l_{\infty}-2 g P C\right) \cosh \chi, \\
\partial_{r}(l \sinh \chi) & =4 g B l \cosh \chi,
\end{aligned}
$$

where we have defined the constant

$$
l_{\infty} \equiv-2\left(k+n C_{0}\right) .
$$

Moreover, straightforward (albeit tedious) computation shows that every solution to Eq. (50) indeed gives a solution to the full set of field equations derived from Eq. (19). For the Abelian Chern-Simons Higgs model (26), these equations reduce to the set of differential equations derived in [15]. In the next section we shall show that for given $n$, these equations admit a unique vortex solution with regular asymptotics.

\section{Asymptotics of the Bogomol'nyi equations}

We are mainly interested in topologically stable, finite energy vortex solutions. In particular, we expect the scalar field $R$ to run from the symmetric AdS vacuum at $r=0, R=0$ into a Minkowski vacuum at $R=\eta$ for $r \rightarrow \infty$. Hence, these solutions cannot be continuously deformed into the vacuum solution.

More precisely, around the origin $r=0$ we assume the following asymptotic AdS behavior of the metric

$$
l=r+\mathcal{O}(r), \quad \chi=m r+\mathcal{O}\left(r^{2}\right),
$$

where the constant $m$ in our conventions is $m=4 g\left(C_{0}^{2}+b\right)$ and gives the inverse AdS radius of the metric. Regularity of the scalar and gauge field around $r=0$ then requires (we assume $n>0, g>0$ )

$$
R=R_{0} r^{n}+\mathcal{O}\left(r^{n+1}\right), \quad P=-\frac{n}{g}+\mathcal{O}\left(r^{2 n}\right),
$$

and fixes $k=-1 / 2$ in Eq. (51). The constant $R_{0}>0$ is the only free parameter in the asymptotics around zero. It will be fixed by demanding regularity of the solution at $r \rightarrow \infty$. For $r \rightarrow \infty$ we assume the following behavior of the matter fields and metric:

$$
\begin{gathered}
R(r) \rightarrow \eta>0, \quad P(r) \rightarrow 0, \\
l(r) \rightarrow l_{\infty} r, \quad \chi(r) \rightarrow \frac{\chi_{\infty}}{r},
\end{gathered}
$$

with constants $l_{\infty}=1-2 n C_{0}$ defined in Eq. (51), and $\chi_{\infty}=n^{2} /\left(2 g l_{\infty}^{2}\right)$. Closer inspection of the differential equations (50) shows that demanding regular asymptotics at infinity leaves one free integration constant which appears in subleading order in $R$, cf. Eq. (67). Asymptotically, the metric (54) describes a locally flat space with deficit angle

$$
\delta=4 n \pi C_{0},
$$

or, more precisely, a particle with mass $M=2 n C_{0}$ and spin $J=n^{2} /(2 g)[1,15,32]$. A well defined conical geometry at radial infinity requires the upper bound

$$
n<\frac{1}{2 C_{0}},
$$

for the vortex number $n$. The values $2 n C_{0}=1$ and $n C_{0}=1$ correspond to cylindrical and spherical asymptotic geometry, respectively [1]. Since Eq. (54) requires the function $B$ as well as the potential $V$ to vanish at radial infinity, i.e. $B(\eta)$ $=V(\eta)=0$, together we find that

$$
C(R=\eta)=0, \quad b=0,
$$

which fixes the constants $b$ and $C_{0}$. Recalling that $C^{\prime}=$ $-2 R G$ and that $G$ remains positive to ensure a nondegenerate kinetic scalar term, this in particular implies that $C_{0}>0$. 
In turn, Eq. (57) already implies that $V^{\prime}(\eta)=0$, i.e. at radial infinity the scalar field runs into a Minkowski vacuum of the potential.

We seek a solution of the system of differential equations (50) which interpolates between the proper asymptotics (52), (53) for $r \rightarrow 0$ and 54 for $r \rightarrow \infty$, respectively. As we have seen, there is precisely one free parameter in the asymptotic expansion around $r=0$. It is a nontrivial problem whether, by properly choosing this parameter $R_{0}$, one may find a set of functions $\{R, P, l, \chi\}$ which obey regular asymptotics (54) at radial infinity also. In the remaining part of this section, we shall show that this is indeed the case.

We start our analysis of the differential equations (50) with some observations. Consider the quantity

$$
Z \equiv P^{2}-4 l \sinh \chi\left(C P-\frac{l_{\infty}}{2 g}\right)-4 l^{2} B .
$$

From the differential equations (50), one may verify that $Z$ is an integral of motion, i.e. $\partial_{r} Z=0$, and hence reduces the number of unknown functions to three. We further observe that Eq. (50) imply the following second order equation for the scalar field:

$$
\frac{\partial}{\partial r}\left(\frac{l \cosh \chi}{R} \frac{\partial R}{\partial r}\right)=8 g^{2} C\left(B-G R^{2}\right) l \cosh \chi .
$$

To analyze the existence of solutions with proper asymptotics at $r \rightarrow 0$ and $r \rightarrow \infty$, we introduce the new radial variable

$$
\tilde{r} \equiv l \cosh \chi R^{-2 C_{0}} e^{K} .
$$

The system of equations (50) then yields the following simple radial equation:

$$
\frac{\partial \ln \tilde{r}}{\partial r}=\frac{l_{\infty}}{l \cosh \chi} .
$$

Recall that the bound for a regular asymptotically conical geometry (56) implies that $0<l_{\infty}<1$ so that Eq. (60) is indeed a well-defined coordinate transformation. In contrast, assuming asymptotically cylindrical geometry corresponds to $l_{\infty}=0$, in which case $\tilde{r}$ is not a well defined coordinate but rather a constant. More precisely, in this case Eq. (61) may be integrated to

$$
l \cosh \chi=\left(R / R_{0}\right)^{1 / n} e^{K(0)-K(R)} .
$$

The first equation of Eq. (50) together with Eq. (58) then reduces to a decoupled first order differential equation for $R$

$$
\partial_{r} R=R \sqrt{n^{2}\left(R / R_{0}\right)^{-2 / n} e^{2[K(R)-K(0)]}+4 g^{2} C^{2}},
$$

which shows that its solution $R$ necessarily diverges at radial infinity. With the ansatz (32)-(34), there are hence no regular solutions with asymptotically cylindrical geometry in these models. This is in agreement with the discussion in [17] for the Abelian Higgs model.

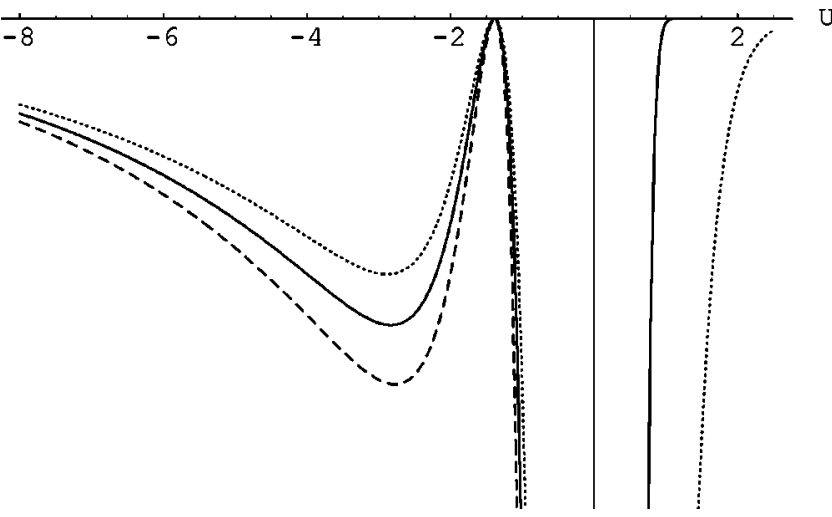

FIG. 3. Effective potential $V_{\text {eff }}$ from Eq. (65) for the potentials $V_{0}$ (solid), $V_{+}$(dotted), $V_{-}$(dashed) from Eqs. (26), (31).

According to Eqs. (52), (54), the new radial variable $\tilde{r}$ (60) has the asymptotic behavior

$$
\begin{aligned}
& \tilde{r} \rightarrow R_{0}^{-2 C_{0}} \cdot r^{l_{\infty}} \quad \text { as } \quad r \rightarrow 0, \\
& \tilde{r} \rightarrow l_{\infty} \eta^{2 C_{0}} e^{K(\eta)} \cdot r \quad \text { as } \quad r \rightarrow \infty .
\end{aligned}
$$

The metric element (34) becomes

$$
\begin{aligned}
& d s^{2}=-(d t-l \sinh \chi d \theta)^{2}+(l \cosh \chi)^{2} \frac{d z d \bar{z}}{z \bar{z}} \\
& \text { with } \quad z=\tilde{r}^{1 / l_{\infty}} e^{i \theta} .
\end{aligned}
$$

The key observation for our analysis, however, is the fact that, in terms of $\tilde{r}$, the second order differential equation (59) completely decouples from the metric functions and takes the form

$$
\frac{1}{\tilde{r}} \frac{\partial}{\partial \tilde{r}}\left(\tilde{r} \frac{\partial U}{\partial \tilde{r}}\right)=-\frac{\partial V_{\mathrm{eff}}}{\partial U}, \quad U=\ln R,
$$

with an effective potential

$$
V_{\mathrm{eff}}=-\frac{2 g^{2}}{l_{\infty}^{2}} e^{4 C_{0} U} e^{-2 K} C^{2} .
$$

The effective potential $V_{\text {eff }}$ is negative definite (as $R>0$ ) and it vanishes at minus infinity and at $U=\ln \eta$, where it has a local maximum since $C(\eta)=0$. For the particular examples given in Eqs. (26) and (31), the effective potential is depicted in Fig. 3.

Note that the gauge coupling constant $g$ may be absorbed by rescaling $\tilde{r}$. As in the case of nongravitating vortices with Higgs potential [22], Eq. (64) can be approximated by a Liouville equation for small $R$ and by a Bessel equation in the vicinity of the Minkowski vacuum at $R=\eta$. More precisely, we find in agreement with Eqs. (53), (54) that 


$$
R \approx\left(2 g C_{0}^{2} e^{-K(0)} \tilde{r}\right)^{-1 / 2 C_{0}}\left[\left(\frac{\tilde{r}_{0}}{\tilde{r}}\right)^{1 / t_{\infty}}-\left(\frac{\tilde{r}}{\tilde{r}_{0}}\right)^{1 / l_{\infty}}\right]^{-1 / 2 C_{0}}
$$

with a constant $\tilde{r}_{0}$ related to $R_{0}$ above, and that

$$
\begin{aligned}
& R \approx \eta+c_{1} K_{0}\left(\frac{2 g}{l_{\infty}} \eta^{2 C_{0}+(1 / 2)} e^{-K(\eta)} C^{\prime}(\eta) \tilde{r}\right) \\
& \quad \text { near } R=\eta
\end{aligned}
$$

where $K_{0}$ denotes the MacDonald function and $c_{1}$ is a constant. Starting either near $R=0$ or near $R=\eta$, the constants $\tilde{r}_{0}$ and $c_{1}$ are implicitly (and uniquely) fixed by requiring regular asymptotics at the other end. Let us now discuss how this comes about in slightly more detail.

The form of Eq. (64) allows us to prove the existence of a regular solution, which interpolates between the proper asymptotics (53) and (54). This may be shown in a manner which is reminiscent of the discussion in [17]. The point is that the second order differential equation (64) can be thought of as describing the damped motion of an auxiliary fictitious particle in the effective potential (65). There is a one-parameter family of solutions which, at $\tilde{r}=0$, start with the correct asymptotics, viz.

$$
U=\frac{n}{l_{\infty}} \log \tilde{r}+\log R_{0}+\cdots
$$

these solutions are labeled by the parameter $R_{0}$ from Eq. (53). Similarly to the analysis in [17], one finds that for small $R_{0}$ these solutions run into the local minimum of the effective potential, whereas for large values of $R_{0}$ they go over the hilltop of the potential at $U=\ln \eta$. There is precisely one value of $R_{0}$ for which the motion of this fictitious particle ends at the local maximum $U=\ln \eta$. This corresponds to the proper asymptotics (54) of the scalar field at radial infinity.

Having solved Eq. (64) with the proper asymptotics at both ends, it remains to restore the other fields $\{P, l, \chi\}$ of the model. To this end, we note that the differential equations (50) imply that

$$
\frac{1}{\tilde{r}} \frac{\partial}{\partial \tilde{r}}(l \sinh \chi)=-\frac{2 l_{\infty}}{g} V_{\mathrm{eff}}
$$

Using Eq. (64), this equation may be integrated to

$$
l \sinh \chi=\frac{l_{\infty}}{2 g}\left[\frac{n^{2}}{l_{\infty}^{2}}-2 \widetilde{r}^{2} V_{\mathrm{eff}}-\left(\tilde{r} \frac{\partial U}{\partial \tilde{r}}\right)^{2}\right],
$$

and one easily verifies that this indeed obeys the correct asymptotics (52), (54). The remaining metric function is obtained from Eq. (60), which gives

$$
l \cosh \chi=\tilde{r} R^{2 C_{0}} e^{-K(R)},
$$

while the gauge field $P$ may be extracted from Eq. (50) as

$$
P=-\frac{l_{\infty}}{g}\left[\tilde{r} \frac{\partial U}{\partial \tilde{r}}+2 \widetilde{r}^{2} C V_{\mathrm{eff}}+C\left(\tilde{r} \frac{\partial U}{\partial \tilde{r}}\right)^{2}-\frac{n^{2}}{l_{\infty}^{2}} C\right] .
$$

All the fields have correct asymptotical behavior (52), (53), (54), provided the function $R$ is the unique solution of Eq. (64) with proper asymptotics. The original radial variable $r$ may finally be restored by integrating (61),

$$
r(\tilde{r})=\frac{1}{l_{\infty}} \int_{0}^{\tilde{r}} d \tilde{r}^{\prime} R^{2 C_{0}} e^{-K(R)} .
$$

To summarize, we have shown that the system of differential equations (50) may be reduced to a single second order differential equation (64) which, for each vortex number $n$ satisfying Eq. (56), admits a unique solution with proper asymptotics at $r=0$ and $r \rightarrow \infty$. From this solution, the original fields may be restored according to Eqs. (70)-(72) and they have the correct asymptotics. This completes the construction of vortex solutions in the general $U(1)$-gauged supergravity (19).

An obstruction to the existence of these vortex solutions may, however, show up for certain compact Kähler manifolds. As has been observed in the four-dimensional case in [29], global consistency of the Lagrangian (19) requires the Kähler manifold to be a Hodge manifold, which may imply a quantization of the gravitational constant in units of the scalar self-coupling. For the compact $S^{2}$ model (29) for example, one finds the restriction

$$
2 C_{0}=\frac{1}{a^{2}} \in \mathbb{Z}
$$

as has been explicitly verified in [18]. This quantization condition is obviously incompatible with the bound (56), and hence reduces the possible values of the vortex number to $n=1$ and $n=2$, corresponding to asymptotically cylindrical and spherical geometry, respectively. As has been discussed above, these solutions do not exist in this model. The absence of vortex solutions with asymptotically conical geometry and the scalar fields living on a compact target manifold has already been noted in four dimensions in [33].

\section{Examples}

Let us illustrate the analysis of Sec. III C by computing some numerical solutions to the specific models presented in Sec. II D. Since the Abelian Chern-Simons Higgs model coupled to gravity (26) and its vortex solutions have already been extensively discussed in the literature [13-17], we will consider the noncompact $H^{2}$ model with potential $V_{-}$given in Eq. (31), whose vortex solutions have not yet been analyzed. In fact, this model may be of special interest, since in the limit $g \rightarrow 0$ it reduces to the theory obtained from dimensional reduction of pure four-dimensional Einstein 


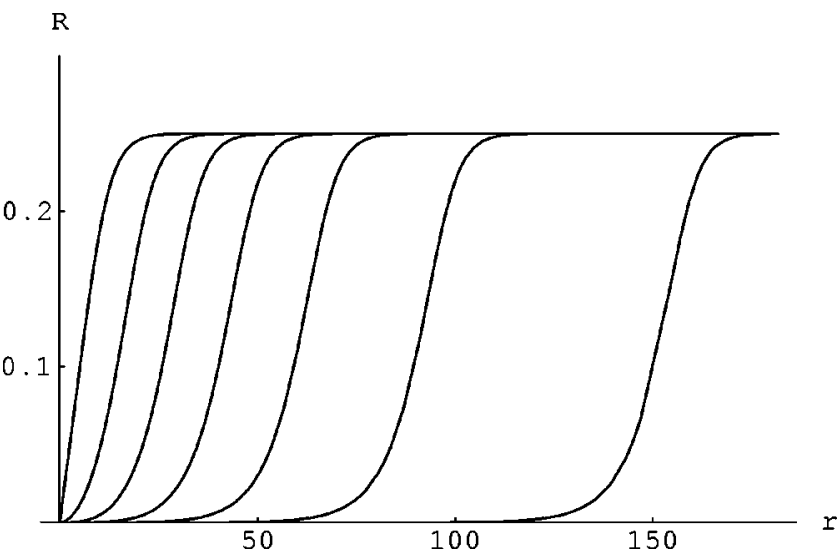

FIG. 4. Scalar field $R(r)$ for vortices in the $H^{2}$ model with $n$ $=1,2, \ldots, 7$, from left to right.

gravity. It is then tempting to speculate about a possible higher-dimensional geometrical origin of these vortex solutions.

For the particular values $a=g=1$ and $\eta=\frac{1}{4}$ of the parameters, the scalar potential $V$ and effective potential $V_{\text {eff }}$ have been depicted in Figs. 1, 2, and 3, respectively. Recall that the choice of a strictly positive $C_{0}$ in this model was essential for the existence of a Minkowski vacuum and hence for the existence of the vortex solutions. With these parameters, Eq. (56) yields the upper bound $n<8$ for the vortex number. For each $0<n<8$, the unique solution $R(\tilde{r})$ to Eq. (64) which has asymptotics (68) around $\tilde{r}=0$ and remains regular at $\tilde{r}=\infty$ may be found numerically, by fine-tuning the unknown parameter $R_{0}$ by hand. We should stress, however, that finding the regular solutions with higher vortex numbers requires considerable numerical accuracy. A nontrivial check is provided by inserting the solution thus obtained into the effective potential $V_{\text {eff }}$ and numerically integrating the righthand side (rhs) of Eq. (69), which should result in

$$
\int_{0}^{\infty} V_{\mathrm{eff}}[R(\tilde{r})] \tilde{r} d \tilde{r}=-\frac{n^{2}}{4 l_{\infty}^{2}},
$$

which is found upon integrating the left-hand side of Eqs. (69) and using Eq. (54). All our numerical solutions pass this check with high precision (up to $0.005 \%$ ). The original radial variable $r$ is finally obtained by numerically integrating Eq. (73). The resulting functions $R(r)$ for all possible values $n$ $=1,2, \ldots, 7$ of the vortex number have been plotted in Fig. 4 , the value of $n$ increasing from left to right. The behavior of the (normalized) gauge field $P(r)$ in these solutions is given in Fig. 5.

\section{SUMMARY AND OUTLOOK}

We have constructed the general three-dimensional $N=2$ gauged supergravities with Abelian gauge group $U(1)$ and Chern-Simons coupling of the vector fields. The supersymmetric Lagrangian and supersymmetry transformations rules were determined up to quartic and cubic fermionic terms, respectively, but we do not expect the structure of the

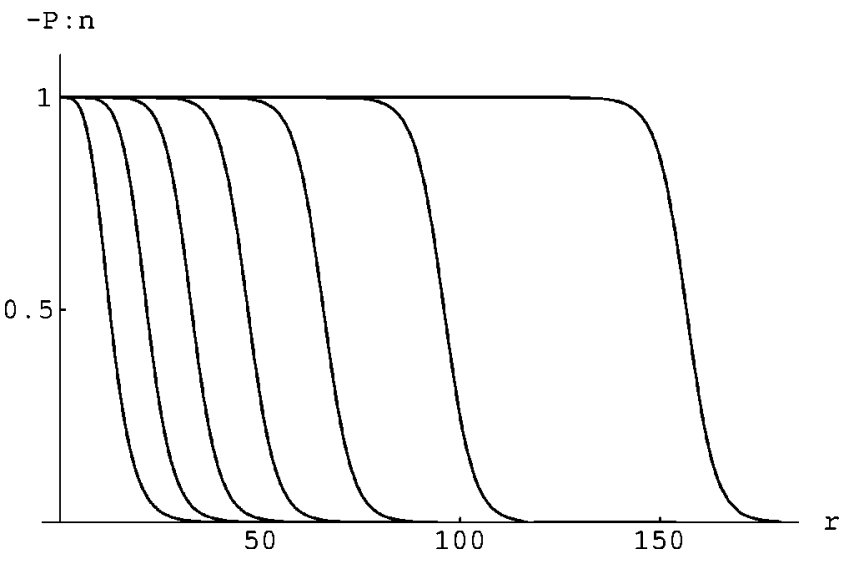

FIG. 5. Gauge field $-P(r) / n$ for vortices in the $H^{2}$ model with $n=1,2, \ldots, 7$, from left to right.

models to be modified by the higher order fermionic terms. The models are parametrized by a Kähler manifold and two real numbers $b$ and $c ; b$ shifts the superpotential for the scalar potential [cf. Eqs. (21) and (22)], while a nonzero value of $c$ corresponds to the presence of a Fayet-Iliopoulos term. The gauged Lagrangians (19) are residually invariant under general Kähler transformations.

We have evaluated our general formulas for various particular examples with a single complex scalar field $\phi$ and Kähler potential $K=K(|\phi|)$. In the case of the complex plane, this reproduces the eighth order polynomial potential (26) of the Abelian Chern-Simons Higgs model coupled to gravity which was previously derived in [13-17] by requiring that the dynamics be given by a system of first order differential equations. This constitutes a natural explanation of these earlier results from local $N=2$ supersymmetry. In particular, our construction provides the embedding of the abelian Chern-Simons Higgs model into a supergravity theory, allowing us to directly address the stability of the vortex solutions preserving half of the supersymmetry. In flat space, the self-dual limit of the Abelian Chern-Simons Higgs model [22] and its particular sixth order potential have similarly been derived from global $N=2$ supersymmetry in [23].

Likewise, for the Kähler manifolds $C P^{p}$ and $C H^{p}$, our results reproduce the potentials of the gauged $N=2$ models studied in [18], together with their generalization by including a Fayet-Iliopoulos term. The presence of this term allows for symmetry-breaking Minkowski vacua of the potentials and hence for vortex solutions.

Having constructed the general Abelian gauged $N=2 \mathrm{su}-$ pergravity theory, we turned to the construction of rotationally symmetric vortex solutions preserving one half of the supersymmetry. Utilizing the ansatz (32)-(34) for the fields, the Killing spinor equations (37) were shown to lead to the system of four first order differential equations (50) (which in particular reduces to the system found in [15] in the special case of the Chern-Simons-Higgs model); it was also verified that this system solves the full set of (second order) field equations of the theory. Furthermore, we showed that this system of equations admits a unique solution in which the norm of the scalar field $R$ runs from the symmetric AdS vacuum of the potential at $r=0, R=0$ into a symmetry 
breaking Minkowski vacuum at $r \rightarrow \infty$. This solution represents a rotationally symmetric, finite energy, topologically stable vortex solution. The essential ingredient for proving the existence of this solution was the further reduction of the system (50) to the single second order differential equation (64). The latter describes the motion of a (fictitious) particle in the effective potential (65). Its solution determines the original fields (scalar, gauge field and components of the metric) via Eqs. (70)-(72).

Our results provide further examples beyond that of Refs. $[25,26]$ of the mechanism proposed in [24] for obtaining a vanishing cosmological constant within a supersymmetric theory without phenomenologically unacceptable BoseFermi degeneracies. In particular, the solution constructed here is the first such example within a gauged supergravity with Abelian Chern-Simons gauge fields (rather than the usual Maxwell fields). The covariantly constant spinors of our solutions exist by virtue of essentially the same mechanism as that pointed out in [25].

Finally, let us mention some directions for further investigations. We have constructed the general Abelian gauged $N=2$ theory, but of course it would be very interesting to obtain non-Abelian gaugings and to identify possible restric- tions on the allowed gauge groups by solving the consistency conditions imposed by local supersymmetry in this case. One would expect such models to admit vortex-type solutions with several gauge fields turned on (i.e. non-Abelian vortices), which it would be interesting to construct explicitly, perhaps by acting with some suitably adjusted solution generating transformations on the Abelian solutions constructed here. The possible relevance of vortex solutions in the AdS/ CFT correspondence has been addressed in [34]. We hope to report on these and related matters in the near future.

\section{ACKNOWLEDGMENTS}

We would like to thank José Edelstein for discussions and collaboration in the initial stages of this work. We also thank the Erwin Schrödinger Institute and the organizers of the program entitled "Mathematical Aspects of String Theory" for hospitality and support in Vienna during its completion. The work of M.A. was supported by the Swiss National Science Foundation under grant number 83EU-056178. This work was supported in part by EU contract HPRN-CT-200000131.
[1] S. Deser, R. Jackiw, and G. 't Hooft, Ann. Phys. (N.Y.) 152, 220 (1984).

[2] S. Giddings, J. Abbott, and K. Kuchar, Gen. Relativ. Gravit. 16, 751 (1984).

[3] E. Witten, Nucl. Phys. B311, 46 (1988).

[4] I. Bengtsson, Phys. Lett. B 220, 51 (1989).

[5] S. Carlip and J.E. Nelson, Phys. Lett. B 324, 299 (1994).

[6] H.-J. Matschull, Class. Quantum Grav. 16, 2599 (1999).

[7] S. Deser, R. Jackiw, and G. 't Hooft, Ann. Phys. (N.Y.) 153, 405 (1984).

[8] S. Deser and J.H. Kay, Phys. Lett. 120B, 97 (1983).

[9] A. Achúcarro and P.K. Townsend, Phys. Lett. B 180, 89 (1986).

[10] B. de Wit, A.K. Tollstén, and H. Nicolai, Nucl. Phys. B392, 3 (1993).

[11] H. Nicolai and H. Samtleben, Phys. Rev. Lett. 86, 1686 (2001).

[12] H. Nicolai and H. Samtleben, J. High Energy Phys. 04, 022 (2001).

[13] P. Valtancoli, Int. J. Mod. Phys. A 7, 4335 (1992).

[14] D. Cangemi and C. Lee, Phys. Rev. D 46, 4768 (1992).

[15] L.A.J. London, Phys. Lett. B 354, 52 (1995).

[16] G. Clément, Phys. Rev. D 54, 1844 (1996).

[17] B.K. Chung, J.-M. Chung, S. Kim, and Y. Kim, Ann. Phys. (N.Y.) 291, 276 (2001).
[18] N.S. Deger, A. Kaya, E. Sezgin, and P. Sundell, Nucl. Phys. B573, 275 (2000).

[19] J. Hong, Y. Kim, and P.Y. Pac, Phys. Rev. Lett. 64, 2230 (1990).

[20] R. Jackiw and E.J. Weinberg, Phys. Rev. Lett. 64, 2234 (1990).

[21] E.B. Bogomol'nyi, Sov. J. Nucl. Phys. 4, 449 (1976).

[22] R. Jackiw, K.-Y. Lee, and E.J. Weinberg, Phys. Rev. D 42, 3488 (1990).

[23] C. Lee, K.-Y. Lee, and E.J. Weinberg, Phys. Lett. B 243, 105 (1990).

[24] E. Witten, Int. J. Mod. Phys. A 10, 1247 (1995).

[25] K. Becker, M. Becker, and A. Strominger, Phys. Rev. D 51, 6603 (1995).

[26] J.D. Edelstein, C. Nuñez, and F.A. Schaposnik, Nucl. Phys. B458, 165 (1996).

[27] J.D. Edelstein, C. Nuñez, and F.A. Schaposnik, Phys. Lett. B 375, 163 (1996).

[28] J.D. Edelstein, Phys. Lett. B 390, 101 (1997).

[29] E. Witten and J. Bagger, Phys. Lett. 115B, 202 (1982).

[30] J.A. Bagger, Nucl. Phys. B211, 302 (1983).

[31] E. Cremmer, S. Ferrara, L. Girardello, and A. Van Proeyen, Nucl. Phys. B212, 413 (1983).

[32] B. Linet, Gen. Relativ. Gravit. 22, 469 (1990).

[33] A. Comtet and G.W. Gibbons, Nucl. Phys. B299, 719 (1988).

[34] M.H. Dehghani, A.M. Ghezelbash, and R.B. Mann, Nucl. Phys. B625, 389 (2002). 